\title{
Caracterización de subsistemas de pasturas braquiarias en hatos de trópico húmedo, Caquetá, Colombia
}

\section{Characterization of sub-systems of Brachiaria grassland in herds from humid tropic, Caquetá, Colombia}

Fecha de recepción: 12 de julio de 2017

Pablo Andrés Motta-Delgado ${ }^{1}$

Fecha de aprobación: 25 de diciembre de 2017

Hernán Eduardo Ocaña-Martínez ${ }^{2}$

DOI: https://doi.org/10.19053/01228420.v15.n1.2018.7759

\section{Resumen}

Se caracterizaron los subsistemas de pasturas braquiarias en hatos de trópico húmedo en el departamento del Caquetá, Colombia. La investigación se realizó en cinco municipios, donde fueron seleccionados 20 agroecosistemas con predominio de pasturas $B$. decumbens o $B$. humidicola, y se desarrolló en dos fases: 1) estática, mediante fichas de caracterización se midieron variables biofísicas del predio, de praderas, de los animales y socio-económicas, y 2) dinámica, se evaluó el desempeño productivo de pasturas y animales. El análisis de variables cualitativas se realizó mediante tablas de contingencia y análisis de correspondencias, y de las cuantitativas, empleando estadística descriptiva y, posteriormente, estadística multivariada (ACP y conglomerados). Los agroecosistemas tienen $83 \pm 11 \%$ del área en pastos, el $100 \%$ cuentan con división y rotación de praderas, con periodos de ocupación de 3,71 $\pm 3,33$ días, el $15 \%$ tienen bancos de proteína, el 85 \% dependen de la actividad ganadera, el 80 \% son manejados por propietarios y en el $10 \%$ realizan enmiendas al suelo. El control de arvenses varió del mecánico, al químico y al mixto. La productividad de materia seca fue 1.825,93 $\pm 905,92 \mathrm{~kg} / \mathrm{ha} / \mathrm{corte}$, capacidad de carga de 0,73 UA/ha, con productividad de leche de 1.492,95 $\pm 416,55 \mathrm{~kg} / \mathrm{ha} / \mathrm{año}$. En conclusión, los agroecosistemas son de agricultura familiar, dedicados al doble propósito, con moderada tecnificación y productividad.

Palabras clave: agroecosistema; Amazonia; doble propósito; medios de vida.

1 Corporación para el Desarrollo Sostenible y Mitigación de Cambio Climático (Florencia- Caquetá, Colombia). ORCID: 0000-0001-8820-5542.

2 M. Sc. Universidad de la Amazonia (Florencia- Caquetá, Colombia). 


\begin{abstract}
This study aims at characterizing the sub-systems of Brachiaria grasslands in humid tropic herds in five municipalities of the department of Caquetá, Colombia. To achieve this, we selected 20 farms with predominance of $B$. decumbes and $B$. humidicola, and divided the study into two phases: 1 ) static, in which we measured the socio-economic and the biophysical variables of the farm, the meadows, and the animals through characterization sheets; and 2) dynamic, in which we evaluated the productive performance of grasslands and animals. The qualitative variables were analyzed by contingence tables and correspondence analysis; while the quantitative variables, by descriptive statistics and, subsequently, multivariate statistics (PCA and clusters). The farms have $83 \pm 11 \%$ of area in grasslands, $100 \%$ divide and rotate the meadows, with periods of occupation of $3.71 \pm 3.33$ days; in addition, $15 \%$ of the farms have protein banks, $85 \%$ depend on livestock activity, $80 \%$ are owner managed, and 10\% amend the soil. Weed control varies among mechanical, chemical, and mixed. The productivity of dry matter was 1,825.93 \pm 905.92 $\mathrm{kg} / \mathrm{ha} /$ court, the grassland load capacity was $0.73 \mathrm{AU} / \mathrm{ha}$, with milk yield of 1,492.95 $\pm 416.55 \mathrm{~kg} / \mathrm{ha} /$ year. In conclusion, the studied farms are dual-purpose family farms, with moderate technification and productivity.
\end{abstract}

Keywords: agroecosystem; Amazonia; dual-purpose cattle; livelihoods.

\title{
Para citar este artículo:
}

Motta-Delgado PA., Ocaña-Martínez HE. Caracterización de subsistemas de pasturas braquiarias en hatos de trópico húmedo, Caquetá, Colombia. Ciencia y Agricultura. 2018; 15(1): 81-92. 


\section{Introducción}

Las pasturas ocupan más del $33 \%$ de las tierras del planeta (1); en Latinoamérica constituyen el principal uso del suelo, en sistemas extensivos de monocultivo, con más del $50 \%$ del área degradada (2). En la Amazonia, el área de pasturas cultivadas es superior a los 90 millones de hectáreas (3), de estas, el departamento del Caquetá aporta más de dos millones $(4,5)$, para el sostenimiento de una población bovina de 1.417.774 cabezas, distribuidas en 22.621 predios, de los cuales el $76 \%$ están enfocados al doble propósito, con predominancia lechera (6). La gestión de las pasturas ha sido la forma más importante de uso del suelo en la Amazonia desde la colonización temprana y aún continúa la expansión en la región (7).

Sin embargo, la gran diversidad de características socioeconómicas y ecológicas que determinan los sistemas de producción agropecuaria a nivel de finca es uno de los principales desafíos que enfrentan los proyectos de desarrollo rural y la generación de tecnologías apropiadas (8). Una adecuada clasificación de los sistemas productivos puede apoyar el diseño de políticas agropecuarias para una zona, facilitar la definición de políticas de transferencia de tecnología y ayudar al conocimiento de la dinámica de desarrollo de una región o al diseño y gestión de proyectos de desarrollo (9). La economía de los agroecosistemas depende en alto grado de los recursos, ciclos y fenómenos de la naturaleza, siendo necesaria la diversidad para amortiguar la variabilidad de fenómenos naturales (10).

El alto grado de heterogeneidad que existe en las explotaciones que conforman una región, dificulta la toma de decisiones de carácter transversal; en tal sentido, agrupar las explotaciones de acuerdo con sus principales diferencias y relaciones busca maximizar la homogeneidad dentro de los grupos y la heterogeneidad entre los grupos (11). Según Contreras y García (12), las metodologías denominadas "investigación de sistemas de finca" enfatizan la comprensión de los sistemas agrícolas tradicionales, como punto de partida, mediante la evaluación en terreno que incluye entrevistas a los productores sobre las características del predio y el análisis de las razones por las cuales se emplean métodos particulares de producción.

El uso de técnicas multivariadas puede ayudar en la toma de decisiones sobre el manejo y uso adecuado del suelo (13). Estudios de caracterización describen las características o variables generales de los agroecosistemas, el ambiente de producción, los recursos alimenticios, el manejo y la salud, entre otros factores (14).

El objetivo del presente artículo fue la caracterización y tipificación de subsistemas de pasturas braquiarias en hatos de trópico húmedo de la Amazonia en el departamento del Caquetá, Colombia.

\section{Materiales y métodos}

\section{A. Ubicación geográfica}

La investigación se desarrolló en el departamento del Caquetá, ubicado al noroeste de la Amazonia colombiana, entre los $00^{\circ} 42^{\prime} 17^{\prime \prime}$ de latitud sur y $02^{\circ} 04^{\prime} 13^{\prime \prime}$ de latitud norte, y los $74^{\circ} 18^{\prime} 39^{\prime \prime}$ y $79^{\circ} 19^{\prime} 35^{\prime \prime}$ de longitud oeste de Greenwich (15). De acuerdo con CORPOICA (16) y García et al. (17), el departamento cuenta con los tres pisos térmicos (frío, templado y cálido); el cálido representa la mayor área del departamento. La precipitación media es de $3.800 \mathrm{~mm} / a n ̃ o$, sin estación seca bien definida (sin embargo, la menor precipitación pluvial se registra generalmente entre los meses de diciembre a enero, y con excesos, entre marzo y noviembre). El índice de erosividad ( $\mathrm{R} \mathrm{o} \mathrm{El}_{30}$ ) multianual es de $2.750 \mathrm{tm} / \mathrm{cm} / \mathrm{h} / \mathrm{h}$, valor tres veces superior a otras regiones del país. La humedad relativa es superior al $80 \%$, pero puede fluctuar entre el 64 al $93 \%$, con temperaturas que oscilan entre 18 y $36{ }^{\circ} \mathrm{C}$, con media anual de $25^{\circ} \mathrm{C}$, característica de un régimen isohipertérmico que genera una evapotranspiración potencial de 1.435 $\mathrm{L} / \mathrm{m}^{2} / a n ̃ o$. La radiación solar media es de 1.800 horas/año, y la intensidad es de $268 \mathrm{cal} / \mathrm{cm}^{2} /$ día, lo que se puede traducir en un potencial de producción de biomasa de 59 t/ha/año. El área de estudio pertenece a una zona de bosque húmedo tropical $(B h T)$, según la clasificación de las zonas de vida de Holdridge. 


\section{B. Selección de los agroecosistemas}

Los agroecosistemas fueron seleccionados de 500 fincas ganaderas menores a 350 ha, distribuidas en nueve municipios, pertenecientes al proyecto Implementación y validación de modelos alternativos de producción ganadera en el departamento del Caquetá, ejecutado por la Gobernación del Caquetá a través de la corporación 'Misión Verde Amazonia'; se consideraron los criterios propuestos por Yamamoto et al. (18), modificados para el presente estudio: a) tamaño de las fincas (30-180 ha), b) fincas con más de ocho vacas en ordeño, c) disponibilidad a cooperar en el proyecto y d) Accesibilidad y vías de comunicación en buen estado.

Los agroecosistemas ganaderos seleccionados para desarrollar el trabajo de investigación fueron 20, ubicados en los municipios de Albania $(n=5)$, San José del Fragua $(n=2)$, La Montañita $(n=4)$, Milán $(n=5)$ y El Paujil $(n=4)$, y fueron divididos en dos grupos: 10 agroecosistemas donde predominan praderas con pasto $B$. humidícola y 10 con predominio de pasto $B$. decumbens.

\section{Recopilación de información}

El proceso de investigación se desarrolló en dos fases: una estática y otra dinámica, teniendo en cuenta la metodología desarrollada por Mejía-Cortez (19). Durante la fase estática se evaluaron las características biofísicas de las fincas: extensión del predio, porcentaje del área en praderas, porcentaje del área en bosque, porcentaje del área en rastrojos, tipo de pasto predominante en las praderas, edad de establecimiento de las praderas, porcentaje de arvenses en praderas, porcentaje de suelo degradado en pradera, características físico-químicas del suelo, porcentaje de cobertura arbórea en praderas, número de bovinos en total, cantidad de vacas de ordeño, crías, novillas, toros y vacas en periodo seco, y los factores socioeconómicos: número de integrantes de la familia, mano de obra ocasional y permanente, tiempo dedicado a la labor de ordeño, animales dedicados a la venta, producción total de leche por día y precio de venta de leche y de queso.
En la fase dinámica fue evaluado el desempeño productivo de las pasturas y las vacas en producción de leche. Mensualmente, durante diez meses, fueron aforadas las pasturas antes del ingreso de las vacas, para estimar producción de biomasa según las metodologías de (20) y (21); se realizó análisis bromatológico, donde se estimó la proteína bruta (digestión Kjeldhal), lignina, Fibra Detergente Neutro (FDN), Fibra Detergente Ácido (FDA) (van Soest) y cenizas (incineración). Dos días después del ingreso de las vacas a las pasturas se realizó pesaje individual de leche, se registró el tipo racial, discriminando en cebuíno, taurino y media sangre (F1), meses en lactancia y número de partos.

Para recopilar la información se procedió a estructurar una ficha tipo encuesta semiestructurada que permitió realizar la caracterización de cada uno de los predios y obtener la información directamente de los propietarios mediante un diagnóstico participativo del productor y la familia.

\section{Análisis de la información}

La información recopilada fue tabulada en una hoja de cálculo, y posteriormente se realizó el procedimiento propuesto por Valerio et al. (11) para la caracterización y tipificación de sistemas ganaderos; fueron seleccionadas variables cuantitativas, empleando estadística descriptiva para determinar las variables con un coeficiente de variación superior al $70 \%$, a las cuales se les realizó tabla de correlación, eliminando una de las variables correlacionadas en más de $65 \%$.

Consecutivamente, se empleó estadística multivariada, siguiendo la metodología abordada por Carrillo et al. (22) para la caracterización y tipificación de sistemas productivos; utilizando análisis de correspondencias múltiples (ACM) para variables cualitativas, siguiendo la metodología trabajada por Cruz y Jaramillo (23), y análisis de componentes principales (ACP), para las cuantitativas, según la metodología trabajada por Coronel y Ortuño (24). Para el análisis estadístico de variables cualitativas se utilizaron tablas de contingencia. El análisis estadístico se desarrolló en el software Infostat versión 2017 (25), que cuenta con interfaz al software estadístico $R$. 


\section{Resultados y discusión}

Fueron recopiladas un total de 121 variables: 70 cuantitativas y 51 cualitativas. De las variables cualitativas, a partir de la frecuencia relativa, fueron seleccionadas como relevantes un total de 10 : pasto predominante, textura del suelo, ¿ $80 \%$ de los ingresos provienen de la ganadería?, persona que maneja la finca, huerta casera, comprador de leche, uso de banco de proteína, enmiendas, cercas eléctricas y tipo de control de arvenses.

Se evidenció que los agroecosistemas ganaderos pertenecen a algún gremio o asociación y son producciones de doble propósito; en todas se encuentra, al menos, un cultivo para autoconsumo; el ordeño es manual y se realiza en horas de la mañana; las praderas cuentan con división en cercas y se rotan con periodos de ocupación de 3,71 $\pm 3,33$ días. De los 20 agroecosistemas, el 15\% tiene bancos de proteína (BP), el $85 \%$ recibe los ingresos generados en un $80 \%$ por la actividad ganadera $(80 \%$ IG), el $25 \%$ posee huertas caseras $(\mathrm{HC})$, el $80 \%$ son manejados por los propietarios (MF), el $10 \%$ realiza enmiendas al suelo de las praderas (ENM), el $80 \%$ cuenta con cercas eléctricas (C.EL), el control de arvenses (CM) es $45 \%$ mecánico, 30\% químico y $25 \%$ mixto; el $60 \%$ de la venta de la leche $(\mathrm{VL})$ se realiza a quesilleras locales, el $30 \%$ a Nestlé, el $5 \%$ es venta mixta (quesilleras-Nestlé) y el $5 \%$ vende queso. El 95\% de los animales destinados a la venta son los terneros destetados, y el $5 \%$ son vacas de descarte. Respecto a la textura de los suelos (TEX), el 55\% es franca; el $35 \%$, franco-arcillosa, y el $10 \%$ franco-arenosa. Las interacciones de las variables cualitativas se muestran en la Figura 1.

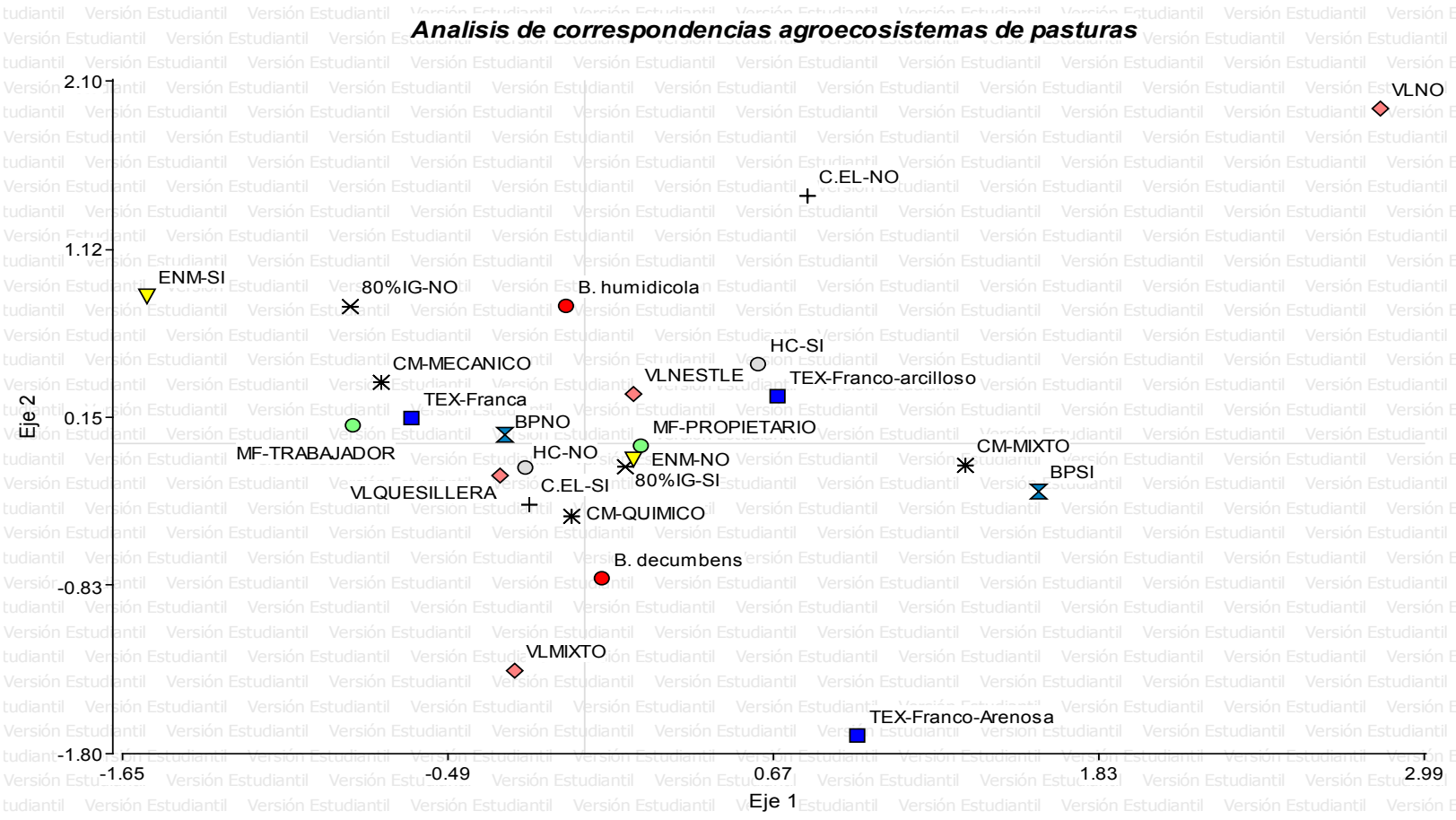

Fig. 1. Análisis de correspondencias múltiples en agroecosistemas ganaderos basados en pasturas del trópico húmedo, Caquetá, Colombia.

No existe una relación estrecha entre el tipo de pasto predominante y las demás variables evaluadas; sin embargo, el control de arvenses en los agroecosistemas con $B$. decumbens está asociado al químico, mientras en agroecosistemas con $B$. humidícola es principalmente mecánico, generalmente con pala, debido a la menor presencia de 
las arvenses, ocasionada por la alta competencia y recuperación de este pasto.

En los agroecosistemas que son manejados directamente por el propietario, el $80 \%$ de los ingresos dependen de la producción ganadera, no se realizan enmiendas y el control de arvenses es químico, y en los agroecosistemas donde el $80 \%$ de los ingresos no dependen de la actividad ganadera, el manejo del predio lo realizan trabajadores agregados (mayordomos) y sí se realizan enmiendas al suelo, por tanto, se puede inferir que hay mayor disponibilidad económica para realizar inversiones.

En los agroecosistemas con presencia de bancos de proteína el control de arvenses es mixto; ade- más, la presencia de huertas caseras (HC-SI) está relacionada con suelos de textura franco-arcillosa, mientras suelos con textura franca no tienen huertas caseras. Es destacable que en los predios con cercas eléctricas hay un estrecho asocio al control químico de arvenses.

Al verificar el coeficiente de variación mayor a $70 \%$ de las variables cuantitativas, solo 18 cumplieron el requisito, y al someterlas a correlación fueron identificadas finalmente seis variables para realizar el análisis: número total de praderas, duración de la labor del ordeño (horas), porcentaje del predio en bosque, número de crías, área de la pradera más pequeña (ha) y el autoconsumo de leche diaria (litros) (Figura 2).

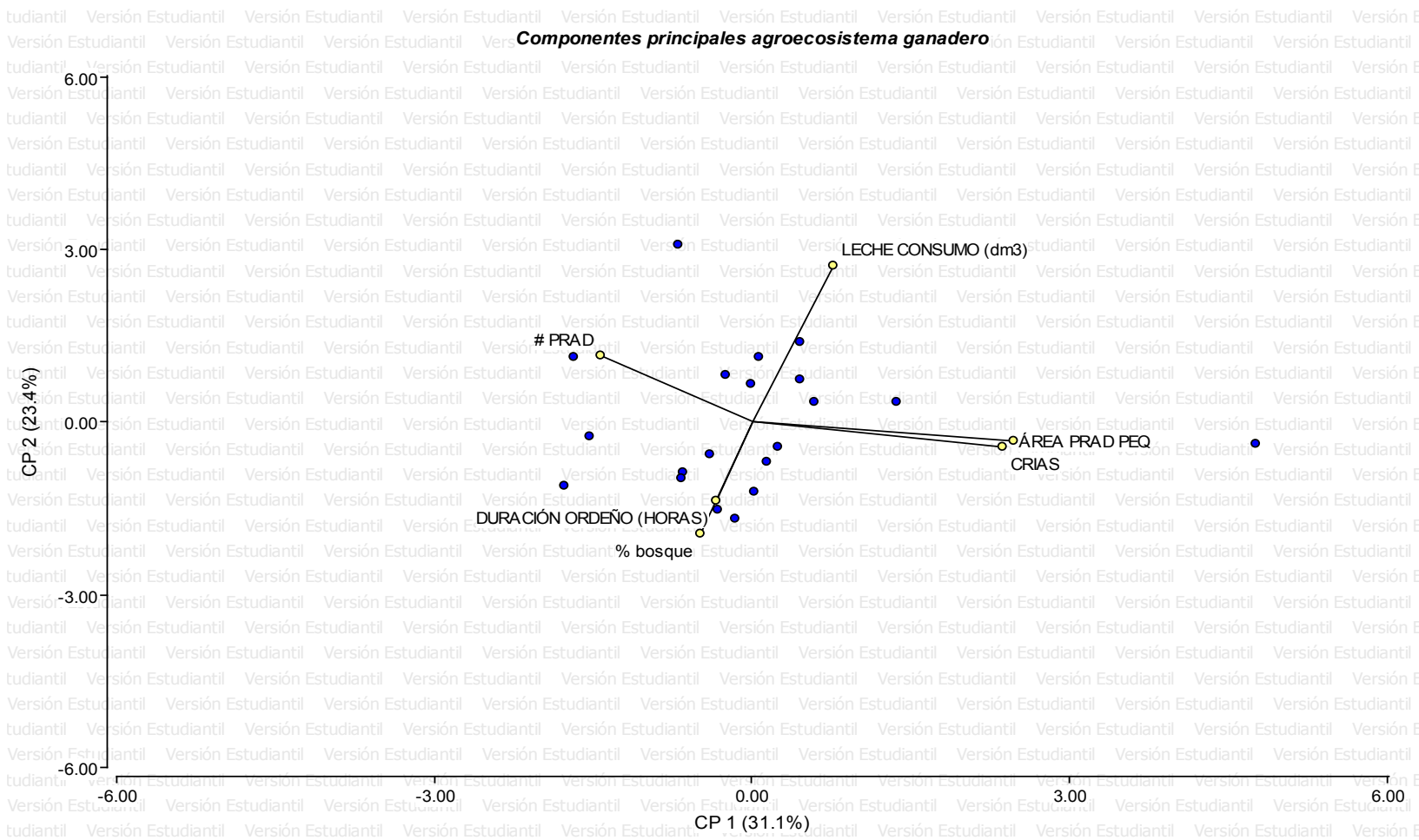

Fig. 2. Análisis de componentes principales en agroecosistemas ganaderos basados en pasturas del trópico húmedo, Caquetá, Colombia.

Es posible inferir, mediante la Figura 2, que a mayor duración de la labor del ordeño del lote de vacas menor es el autoconsumo de leche, lo cual se debe, principalmente, a que los predios de mayor cantidad de vacas en ordeño, y, por ende, mayor producción de leche, son generalmente administrados por mayordomos, mientras que en predios de baja producción, mayor cantidad de leche es destinada al autoconsumo de la familia. 
De igual manera, se encontró que al incrementar la cantidad de praderas pequeñas existe una fuerte correlación positiva con el incremento del número de crías y, por tanto, de vacas en producción de leche mantenidas en el predio, lo cual es indicador de mayor eficiencia en el aprovechamiento de las pasturas y del mejoramiento en la productividad del área.
Se realizó tipificación de los agroecosistemas por medio de conglomerados (Figura 3) con dos grupos mediante el método de promedio (Average linkage) y distancia Euclidea, obteniéndose que no hay diferencia estadística en los grupos con un punto de corte de 5,18 , que equivale al $50 \%$ de la distancia, como lo recomienda (25). La correlación cofonética es alta, siendo del 0,865.

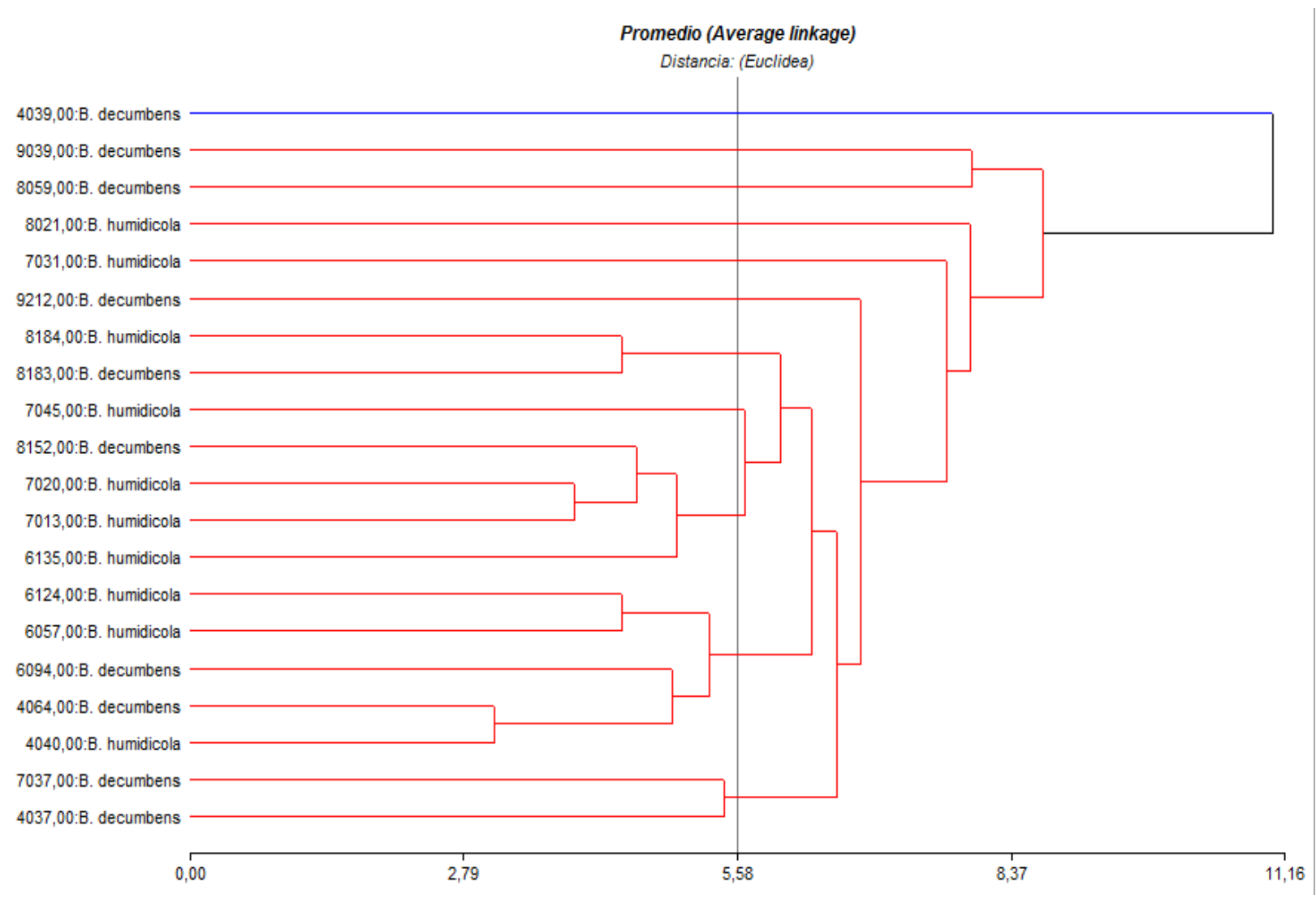

Fig. 3. Tipificación por conglomerados de subsistemas de pasturas en predios de trópico húmedo, Caquetá, Colombia.

Teniendo en cuenta que no se halló diferencia estadística significativa para la formación de dos grupos conglomerados con punto de corte mínimo de 5,58, el análisis de las variables se realizó mediante estadística descriptiva para todos los casos analizados como un solo grupo (Tabla I). 
Tabla I. Estadística descriptiva de variables en agroecosistemas de pasturas en hatos del trópico húmedo, Caquetá, Colombia.

\begin{tabular}{|c|c|c|c|c|c|c|}
\hline Variable & $\mathrm{n}$ & Media & D.E. & $\mathrm{CV}$ & Mín. & Máx. \\
\hline Área predio & 20 & 64,70 & 28,13 & 43,48 & 25,00 & 126,00 \\
\hline$\%$ pastos & 20 & 83 & 11 & 13,55 & 56 & 100 \\
\hline \% bosque & 20 & 12 & 10 & 85,57 & 0,00 & 37 \\
\hline$\%$ rastrojo & 20 & 4 & 4 & 99,47 & 0,00 & 15 \\
\hline$\%$ otros & 20 & 1 & 1 & 116,77 & 0,00 & 3 \\
\hline Número praderas & 20 & 17,10 & 9,88 & 57,76 & 5,00 & 40,00 \\
\hline Área pradera grande (ha) & 20 & 5,15 & 3,72 & 72,18 & 1,00 & 17,00 \\
\hline Área pradera pequeña (ha) & 20 & 1,88 & 2,48 & 132,03 & 0,50 & 12,00 \\
\hline Ocupación pradera (días) & 17 & 3,71 & 3,33 & 89,88 & 1,00 & 15,00 \\
\hline Número fuentes agua & 20 & 6,30 & 4,34 & 68,92 & 2,00 & 19,00 \\
\hline Densidad aparente suelo & 20 & 1,12 & 0,06 & 5,04 & 1,01 & 1,23 \\
\hline Biomasa kg MS/ha/corte & 91 & 1825,93 & 905,92 & 49,61 & 436,8 & 4452,8 \\
\hline Altura pasto $(\mathrm{cm})$ & 32 & 26,66 & 7,01 & 26,29 & 14,10 & 44,90 \\
\hline Proteína bruta (\%) & 87 & $6,59 \%$ & 1,52 & 23,02 & 4,00 & 11,50 \\
\hline Lignina $(\%)$ & 87 & 12,31 & 3,66 & 29,70 & 3,84 & 24,22 \\
\hline FDN (\%) & 87 & 81,61 & 4,77 & 5,85 & 68,74 & 89,56 \\
\hline FDA (\%) & 87 & 46,32 & 4,53 & 9,78 & 31,09 & 55,77 \\
\hline Cenizas (\%) & 87 & 9,15 & 1,67 & 18,24 & 5,96 & 12,82 \\
\hline Personas familia & 20 & 5,70 & 1,63 & 28,52 & 3,00 & 10,00 \\
\hline Personas dependen finca & 20 & 4,95 & 1,54 & 31,07 & 2,00 & 8,00 \\
\hline Cabezas ganado & 20 & 56,30 & 24,79 & 44,03 & 25,00 & 100,00 \\
\hline Capacidad de carga UA/ha & 20 & 0,73 & 0,28 & 38,58 & 0,26 & 1,60 \\
\hline Vacas ordeño & 20 & 16,50 & 8,37 & 50,73 & 6,00 & 40,00 \\
\hline Total leche/día (kg) & 20 & 103,50 & 68,21 & 65,90 & 25,00 & 335,00 \\
\hline Leche/vaca/día (kg) & 20 & 6,08 & 1,46 & 23,98 & 4,12 & 9,43 \\
\hline Leche/ha/año (kg) & 20 & $1.492,95$ & 416,55 & 27,9 & 1014 & 2385 \\
\hline Duración ordeño (horas) & 20 & 1,63 & 0,86 & 52,71 & 1,00 & 4,00 \\
\hline Leche consumo (litros) & 20 & 1,85 & 1,04 & 56,22 & 0,00 & 4,00 \\
\hline Valor litro leche (COP\$) & 19 & 993,68 & 92,69 & 9,33 & 850,00 & 1200,00 \\
\hline Ingresos leche/ha/año (COP\$) & 20 & 754.916 & 396.359 & 52,50 & 160.088 & 1.746 .786 \\
\hline Edad destete (meses) & 20 & 9,15 & 1,42 & 15,57 & 7,00 & 12,00 \\
\hline Peso al destete $(\mathrm{kg})$ & 20 & 168,5 & 30,14 & 17,88 & 130 & 270 \\
\hline Peso venta/animal (kg) & 18 & 167,22 & 81,37 & 48,66 & 0,00 & 450,00 \\
\hline Edad venta (años) & 17 & 1,16 & 1,77 & 152,57 & 0,58 & 8,00 \\
\hline
\end{tabular}

n: casos observados, DE: desviación estándar, CV: coeficiente de variación, Mín.: valor mínimo, Máx.: valor máximo, MS: materia seca, FDN: fibra detergente neutro, FDA: fibra detergente ácido. COP\$: pesos colombianos, UA/ha: unidades animal por hectárea, igual a $450 \mathrm{~kg}$ peso vivo.

Los agroecosistemas del estudio se ubican dentro de las condiciones de la unidad agrícola familiar (UAF) establecida para el departamento del Caquetá (260,59 ha), según el acuerdo 140 de 2008 (26), y por tanto se pueden catalogar como explotaciones de agricultura familiar según las consideracio- nes de FAO (27). De acuerdo con FAO-FEPALE (28), las fincas son medianas, porque producen más de 100 litros de leche al día, pero según Pallares (29) son pequeñas pues tienen menos de 80 cabezas de ganado. En tal sentido, al cumplir dos de tres criterios de clasificación, los agroecosistemas 
ganaderos evaluados son explotaciones de agricultura familiar.

Ocaña-Martínez (30) halló que la ganadería tradicional en el Caquetá, donde predominan pastos en monocultivo, representa el $65,1 \%$ de los predios; el área predial promedio fue de 132 ha, con mínimo y máximo de 25 y 352 ha, respectivamente, valor promedio que dobla el hallado en este estudio; sin embargo, hay coincidencia en el valor mínimo de área. Además, reporta que el área en pastos es cercana al $80 \%$, valor coincidente con el de este estudio y con el $82 \%$ y $81 \%$ reportados por Rivas y Holman (31) y Velásquez et al. (32); no obstante, difieren del reporte de Pallares (29), para quien las áreas de pastoreo son menores al $60 \%$.

Pallares (29) refiere que la capacidad de carga en el departamento del Caquetá es de 0,8 cabezas, y que en el $99 \%$ de los predios el sistema de ordeño es manual, datos similares a los encontrados aquí. Además, Ocaña-Martínez (30) reporta que $B$. decumbens y $B$. humidícola son las especies de pastos mejorados más utilizados en predios del Caquetá, donde el $48 \%$ de los encuestados reportaron más de dos especies de pastos en sus predios, reporte que coincide con lo aquí observado, no obstante, con predominio de una especie.

Medina-Gutiérrez (33), en un estudio realizado en la cuenca hidrográfica del río San Pedro, en San José del Fragua, Caquetá, hallaron que el uso principal del suelo es la ganadería extensiva de doble propósito, donde las pasturas predominantes son las Brachiarias: $B$. decumbens y $B$. brizantha $\mathrm{CV}$ Marandú; dicha cuenca abarca las fincas incluidas en este estudio, por tanto, los resultados son coincidentes. No obstante, reportan baja productividad, que no concuerda con los resultados de la presente investigación. Así mismo, reportan áreas promedio de las praderas de cuatro hectáreas, que son inferiores a las áreas de praderas grandes aquí halladas. Además, se reporta el uso de herbicidas, lo cual coincide con el control químico que realizan los productores en agroecosistemas con predominio de $B$. decumbens. Los cultivos de pancoger en la cuenca representan el $2 \%$, cifra superior a la de otros de la presente investigación, que fue del $1 \%$. Los rastrojos representaron el $5 \%$, superior al $4 \%$ hallado en esta investigación.

En el municipio de Solita, Caquetá, la principal actividad económica es la producción bovina de doble propósito, en predios con áreas de pasturas que varían entre las 60 ha y 200 ha, donde destacan pasturas del género Brachiaria, para el sostenimiento de 50 a 150 semovientes (34), datos coincidentes a los hallados en esta investigación.

En explotaciones lecheras en Centroamérica fue reportada baja tecnificación y hatos pequeños con baja carga animal, por lo que son consideradas de baja intensidad productiva (35), resultados similares a la presente investigación, donde el nivel tecnológico de los predios es bajo, con escasa inversión en las praderas y cargas animales inferiores a $0,73 \mathrm{UA} / \mathrm{ha}$; la baja intensidad productiva en Centroamérica se debe al predominio de animales de mestizaje indefinido (35), que coincide con las características de los predios analizados, en donde el tipo de explotación es de doble propósito, con animales mestizos sin biotipo racial definido. De igual manera, reportan una producción de leche y productividad por animal de $60,1 \mathrm{~kg}$ y $5,8 \mathrm{~kg}$, respectivamente, inferior a los resultados aquí reportados.

La ganadería en el trópico, y especialmente en América Latina, se ha caracterizado por una baja eficiencia productiva (35). En hatos DP en México fueron reportados predios con extensión promedio de 26,2 $\pm 41,93$ ha, porcentaje de área de pasto cercana al $100 \%$, con producción de leche diaria de 53,5 litros, con media por vaca de 4,48 $\pm 1,82$ litros (36), valores estos inferiores a los hallados en la presente investigación. Así mismo, reportaron edad al destete de 11 meses y peso promedio de $190 \mathrm{~kg}$, mayores a los 9,15 meses y 167,22 kg encontrados aquí.

Según un estudio en predios con tecnificación intermedia $(n=17)$ en el estado de Veracruz, México, el área promedio de predios fue de $85,1 \pm 23,8$ ha, capacidad de carga de $1,0 \pm 0,3 \mathrm{UA} /$ ha y producción de $611,8 \pm 175,2$ I/ha/año (37); es decir, pese a tener mejor capacidad de carga, la productividad por hectárea fue menor a lo encontrado en esta 
investigación. En el estado de Michoacán, México, la productividad por vaca fue de 9,2 I/día, y total del hato de 64 I/día (38), siendo una productividad por vaca mayor a la aquí encontrada.

En el estado de Veracruz, México, reportan que entre el $65,2 \%$ y el $67 \%$ de los productores dependen económicamente de la actividad ganadera doble propósito (35) y (39), contrastando con el $85 \%$ de dependencia hallado en la presente investigación. A nivel de pasturas, en el estado de Veracruz fue reportado que las praderas están conformadas por pastos nativos y mejorados (48 $\%)$, exclusivo pastos nativos (26\%) y con pastos mejorados $(26 \%)$, con sistemas de pastoreo rotacional $(64 \%)(40)$, mientras que en el $100 \%$ de los agroecosistemas del presente estudio hay predominio de pasturas mejoradas del género Brachiaria y menor proporción de praderas destinadas a las crías con pastos naturalizados y $100 \%$ de división de rotación de praderas con periodos de ocupación superior a las recomendaciones zootécnicas y agronómicas.

En algunas lecherías de Chile la leche no es enfriada, y los productores tienen dedicación exclusiva a la actividad ganadera (22); para el caso del departamento del Caquetá, el $30 \%$ de los predios evaluados venden la leche refrigerada a Nestlé, y el 85 $\%$ se dedica totalmente a la actividad ganadera y el $15 \%$ a otras actividades.

\section{Conclusiones}

Los agroecosistemas evaluados cuentan con el subsistema de pasturas braquiarias como el principal uso del suelo y presentan características para clasificarlos como explotaciones de agricultura familiar, con alta dependencia de la actividad ganadera basada en la producción de leche en sistemas de doble propósito, con tecnificación y productividad moderada, teniendo en cuenta las condiciones edafoclimáticas y zootécnicas de la región. Además, pese a la moderada tecnificación del sistema, la inversión en las pasturas basada en enmiendas y fertilización es escasa; se basa en un modelo principalmente extractivista, con baja capacidad de carga animal y moderada compactación de los suelos.

\section{Agradecimientos}

El autor principal agradece a la gobernación del departamento del Huila por la financiación de sus estudios de maestría, a través de la convocatoria 677 de 2014; así mismo, agradece a Misión Verde Amazonia, que brindó el espacio, en el marco del proyecto Implementación y validación de modelos alternativos de producción ganadera en el departamento del Caquetá, para el desarrollo de la presente investigación.

Los autores manifiestan que no existen conflictos de intereses en el desarrollo de esta investigación y que han contribuido equitativamente a la elaboración del artículo.

\section{Referencias}

(1) Jose S., Walter D., Mohan-Kumar B. Ecological considerations in sustainable silvopasture desing and management. Agroforestry Systems. 2017; 1-15. DOI: http://doi.org/10.1007/s10457-0160065-2.

(2) Sepúlveda C., Ibrahim M., Bach O., Rodríguez A. Desarrollo de lineamientos para la certificación de sistemas sostenibles de producción ganadera. Agroforestería de las Américas. 2011; 48: 14-20.

(3) Silva RdO., Barioni LG., Hall JAJ., Moretti AC., Veloso RF., Alexander P., et al. Sustainable intensification of Brazilian livestock production through optimized pasture restoration. Agriculural Systems. 2017; 153: 201-211. DOI: http://doi.org/10.1016/j. agsy.2017.02.001.

(4) Beltrán-Barreiro Y., Torrijos RR. Línea base de la industria láctea del Caquetá. Florencia; 2013.

(5) Murcia U., Medina R., Rodríguez JM., Castellanos H., Hernán$\operatorname{dez}$ A., Herrera E. Monitoreo de los bosques y otras coberturas de la Amazonia colombiana, a escala 1:100.000. Datos del periodo 2012, Bogotá, D.C.: Instituto Amazónico de Investigaciones Científicas, Sinchi; 2014.

(6) Caquetá. Consolidado Pecuario EVA 2016. Florencia; 2016.

(7) Numata I., Roberts DA., Chadwick OA., Schimel J., Sampaio FR., Leonidas FC., et al. Characterization of pasture biophysical properties and the impact of grazing intensity using remotely sensed data. Remote sensing of Environment. 2007; 109: 314-327. DOI: http://doi.org/10.1016/j.rse.2007.01.013.

(8) Sánchez-Olaya DM., Suárez-Salazar JC. Caracterización de fincas con arreglos agroforestales de cacao en el Bajo Caguán, departamento del Caquetá (Colombia). In Suárez-Salazar JC. Manejo de arreglos agroforestales de cacao en la Amazonia Colombiana. Florencia: Universidad de la Amazonia; 2014.

(9) Coronel RM., Pérez OSF. Tipificación de los sistemas productivos agropecuarios en el área de riego de Santiago del Estero, 
Argentina. Problemas del desarrollo. Revista Lationoamericana de Economía. 2005; 36(140): 63-88.

(10) Cruz S., Torres G. Caracterización del agroecosistema cafetalero en la Sierra Sur de Oaxaca. In Pérez-Soto F., Figueroa-Hernández E., Godínez-Montoya L., Quiróz-Rocha J., García-Núñez RM. Química, Biología y Agronomía. Texcoco de Mora: ECORFAN; 2016.

(11) Valerio CD., García MA., Acero CR., Cataldo A., Perea JM., Martos PJ. Metodología para la caracterización y tipificación de sistemas ganaderos. Documentos de trabajo producción animal y gestión. 2004.

(12) Contreras LS., García BS. Socioeconomic characterization the production system Potato in the province of Barranca. Big Bang Faustiniano. 2016; 5(2): 37-41.

(13) Oliveira IA., Campos MCC., Freitas L., Soares MDR. Caracterização de solos sob diferentes usos na região sul do Amazonas. Acta Amazónica. 2015; 45(1): 1-12. DOl: http://doi. org/10.1590/1809-4392201400555.

(14) Magaña JG., Parra-Bracamonte GM., Estrada-León RJ., Ku-Vera JC., Sosa-Ferreyra CF. Characterization of animal genetic resource in the desing of sustainable cattle production systems in the tropics. Tropical and Subtropical Agroecosystems. 2009; 10: 8594.

(15) Departamento del Caquetá. Plan de desarrollo 2012-2015: Gobierno de oportunidades. Florencia, Caquetá: Gobernación de Caquetá. 2012.

(16) Corpoica. Aspectos de los suelos del departamento del Caquetá con relación al uso y manejo. Florencia: Corporación Colombiana de Investigación Agropecuaria (Corpoica); 1998.

(17) García J., Cipagauta M., Gómez J., Gutiérrez A. Descripción, especialización y dinámica de los sistemas de producción agropecuaria en el área intervenida del departamento de Caquetá. Florencia, Caquetá: Corporación Colombiana de Investigación Agropecuaria (Corpoica); 2002.

(18) Yamamoto W., Dewi IA., Ibrahim M. Effects of silvopastoral areas on milk production at dual-purpose cattle farms ar the semi-humid old agricultural frontier in central Nicaragua. Agricultural Systems. 2007; 94: 368-375. DOI: http://doi.org/10.1016/j. agsy.2006.10.011.

(19) Mejía-Cortez NA. Evaluación del componente bovino en sistemas de doble propósito manejado bajo condiciones agrosilvopastoriles en Jutiapa, Guatemala. Tesis de Maestría. Turrialba, Costa Rica; 1993.

(20) Maldonado G., Velásquez JE. Determinación de la capacidad de carga y la ganancia de peso de bovinos en pastoreo de gramíneas nativas en el Piedemonte amazónico de Colombia. Pasturas Tropicales. 1994; 16(2): 2-8.

(21) Herrera RS. Toma y procesamiento de la muestra de pasto. Su influencia en indicadores morfológicos y composición química. Revista Cubana de Ciencia Agrícola. 2007; 41(3): 209-216.

(22) Carrillo LB., Moreira VH., González VJ. Caracterización y tipificación de sistemas productivos de leche en la zona centro-sur de Chile: un análisis multivariable. IDESIA. 2011; 29(1): 71-81. DOI: http://doi.org/10.4067/S0718-34292011000100010.
(23) Cruz CGN., Jaramillo BCl. Caracterización y tipificación de sistemas de producción orgánica en la región del Sumapaz. Trabajo de grado. Bogotá D.C.: Fundación Universitaria Los Liberadores, Departamento de Ciencias Básicas; 2016.

(24) Coronel RM., Ortuño PSF. Tipificación de sistemas productivos agropecuarios en el área de riego de Santiago del Estero, Argentina. Problemas del Desarrollo. Revista Latinoamericana de Economía. 2005; 36(140): 63-88.

(25) Di Rienzo JA., Casanoves F., Balzarini MG., González L., Tablada M., Robledo CW. Infostat versión 2017. Córdoba: Universidad Nacional de Córdoba; 2017.

(26) Colombia - INCODER. Acuerdo 140. 2008, mayo 7. (Boletín oficial del estado Número 47122 del 24 de septiembre de 2008).

(27) Salcedo S., Guzmán L. Agricultura familiar en América Latina y el Caribe. Recomendaciones de política. Primera ed. Salcedo S, Guzmán L. (editores). Santiago, Chile: FAO - Organización de las Naciones Unidas para la Alimentación y la Agricultura; 2014.

(28) FAO; FEPALE. Situación de la lechería en América Latina y el Caribe en 2011, Santiago de Chile: FAO; 2012.

(29) Pallares-Villegas Z. Caracterización integral de la cadena de valor del sector lácteo en: Valle de Ubaté-Chiquinquirá y departamento del Caquetá. Informe final de proyecto. Bogotá, D.C: Propaís; 2014.

(30) Ocaña-Martínez HE. Caracterización socioeconómica de tres sistemas de producción en el piedemonte Amazónico Colombiano. Tesis de maestría. Florencia, Caquetá: Universidad de la Amazonia, Facultad de Ciencias Agropecuarias; 2010.

(31) Rivas L., Holman F. Adopción temprana de Arachis Pintoi en el trópico húmedo: el caso de los sistemas ganaderos de doble propósito en Caquetá, Colombia. Pasturas Tropicales. 1999; 21(1): 2-17.

(32) Velásquez JE., Cipagauta HM., Gómez MJE., Tapia CMN. Avances en la caracterización estática de las empresas ganaderas de doble propósito del piedemonte amazónico colombiano. En V encuentro de investigadores del piedemonte amazónico. Mocoa, Putumayo. 2000.

(33) Medina-Gutiérrez G. Caracterización y análisis de usos del suelo en la cuenca del río San Pedro en la zona con función amortiguadora del parque Nacional Natural Alto Fragua Indi Wasi, Municipio de San José del Fragua, Caquetá. Proyecto Aplicado. Florencia, Caquetá: Universidad Nacional Abierta y a Distancia (UNAD); 2017.

(34) Loaiza-Aldana R., Loaiza-Aldana S. Evaluar la aplicación de la metodología diagnóstico y diseño agroforestal (D\&D) en fincas del municipio de Solita - departamento del Caquetá. Trabajo de grado. Solita, Caquetá: Universidad Nacional Abierta y a Distancia (UNAD), Escuela de Ciencias Agrícolas, Pecuarias y del Medioambiente; 2016.

(35) García K., Abarca S., Vargas B., Zúñiga G. Caracterización de los sistemas lecheros en San Joaquín de Tuís, Turrialba, Costa Rica. Revista de Investigación. 2016; 1(2): 45-51.

(36) Orantes-Zebadúa MA., Platas-Rosado D., Córdova-Avalos V., Santos-Lara M., Córdoba-Avalos A. Caracterización de la gana- 
dería doble propósito en una región de Chiapas, México. Ecosistemas y Recursos Agropecuarios. 2014; 1(1): 49-58.

(37) Oros-Noyola V., Díaz-Rivera P., Vilaboa-Arroniz J., Martínez-Dávila JP., Torres-Hernández G. Caracterización por grupos tecnológicos de los hatos ganaderos doble propósito en el municipio de las Choapas, Veracruz, México. Revista Científica. 2011; 21(1): 57-63.

(38) Villa-Méndez Cl., Tena MJ., Tzintzun R., Val D. Caracterización de los sistemas ganaderos en dos comunidades del municipio de Tuzantla de la región de Tierra Caliente, Michoacán. Avances en Investigación Agropecuaria. 2008; 12(2): 45-58.

(39) Vilaboa-Arroniz J., Díaz-Rivera P., Ruiz-Rosado O., Platas-Rosado DE., González-Muñoz S., Juárez-Lagunes F. Caracterización socioeconómica y tecnológica de los agroecosistemas de doble propósito de la región del Papaloapan, Veracruz, México. Tropical and Subtropical Agroecosystems. 2009; 10(1): 53-62.

(40) Vilaboa-Arroniz J., Díaz-Rivera P. Caracterización socioeconómica y tecnológica de los sistemas ganaderos en siete municipios del estado de Veracruz, México. Zootecnia Tropical. 2009; 27(4): 427-436. 